\title{
Electrical LeaderRank Method for Node Importance Evaluation of Power Grids Considering uncertainties of Renewable Energy
}

\author{
Zhou, Bin; Lei, Yifei; Li, Canbing; Fang, Baling; Wu, Qiuwei; Li, Lijuan; Li, Zhenqiu
}

Published in:

International Journal of Electrical Power \& Energy Systems

Link to article, DOI:

10.1016/j.ijepes.2018.09.034

Publication date:

2018

Document Version

Peer reviewed version

Link back to DTU Orbit

Citation (APA):

Zhou, B., Lei, Y., Li, C., Fang, B., Wu, Q., Li, L., \& Li, Z. (2018). Electrical LeaderRank Method for Node Importance Evaluation of Power Grids Considering uncertainties of Renewable Energy. International Journal of Electrical Power \& Energy Systems, 106, 45-55. https://doi.org/10.1016/j.ijepes.2018.09.034

\section{General rights}

Copyright and moral rights for the publications made accessible in the public portal are retained by the authors and/or other copyright owners and it is a condition of accessing publications that users recognise and abide by the legal requirements associated with these rights.

- Users may download and print one copy of any publication from the public portal for the purpose of private study or research.

- You may not further distribute the material or use it for any profit-making activity or commercial gain

- You may freely distribute the URL identifying the publication in the public portal 


\section{Abstract}

\section{Highlights}

\title{
Electrical LeaderRank Method for Node Importance Evaluation of Power Grids Considering Uncertainties of Renewable Energy
}

\author{
Bin Zhou ${ }^{\mathrm{a}, \mathrm{b} *}$, Yifei Lei ${ }^{\mathrm{a}, \mathrm{b}}$, Canbing $\mathrm{Li}^{\mathrm{a}, \mathrm{b}}$, Baling Fang ${ }^{\mathrm{c} *}$, Qiuwei $\mathrm{Wu}^{\mathrm{d}}$, Lijuan $\mathrm{Li}^{\mathrm{a}}$, Zhenqiu $\mathrm{Li}^{\mathrm{a}}$ \\ ${ }^{a}$ College of Electrical and Information Engineering, Hunan University, Changsha 410082, China \\ ${ }^{\mathrm{b}}$ Hunan Key Laboratory of Intelligent Information Analysis and Integrated Optimization for Energy Internet, \\ Hunan University, Changsha 410082, China \\ ${ }^{c}$ College of Electrical and Information Engineering, Hunan University of Technology, Zhuzhou, 412007, China \\ ${ }^{\mathrm{d} D e p a r t m e n t}$ of Electrical Engineering, Technical University of Denmark, Lyngby 2800, Denmark
}

Node importance evaluation of power grids plays an important role in the safe operation and planning of power systems. This paper proposes an electrical LeaderRank (ELR) method to identify the important nodes in complex power grids, considering the renewable energy uncertainties, system topological structure, transmission power flow and the loss of loads caused by cascading failures. The proposed method uses an ELR value function to represent the importance of a system node, which can be derived iteratively from the weighted distribution strategy of its in-linked nodes. Furthermore, the uncertainties of wind and solar energy generation are modelled as interval numbers, and the interval power flow calculation is employed to obtain the interval power in transmission lines, direct adjacent matrix, and finally the interval ELR values. The performance of ELR method has been fully tested and benchmarked on the IEEE 118-bus power system and the Western Liaoning Power Grid of China. Comparative results on four performance criteria have not only demonstrated the validity and superiority of the proposed method, but also confirmed its capability to cope with the node importance evaluation of practical power grids.

An electrical LeaderRank (ELR) method is proposed to identify the important nodes in power grids.

The uncertainties of renewable energy sources are considered in the node importance evaluation.

An interval DC power flow based cascading failure model of power grids is developed.

The weighted rules of ELR values are formulated based on interval power flow distributions.

Keywords: Cascading failures, complex network, photovoltaics, wind energy, vulnerability.

\footnotetext{
*E-mail addresses: binzhou@hnu.edu.cn (B. Zhou), baitin.fong@gmail.com (B. Fang).
} 
The power grid is a complex network threatened by natural disasters, deliberate attacks and equipment failures, and a single disturbance or contingency may trigger a chain effect of unpredictable and disastrous consequences [1]. The failure of important nodes in power grids would cause large-scale power blackouts, and may impact the economy and national security. Therefore, the system important nodes should be identified for power system reinforcement, relay protection, as well as power grid resilience enhancement for the prevention of load interruptions and protection of life and property from the consequences of electricity outages [2],[3]. As an important part in the vulnerability evaluation of power grids, the node importance evaluation can provide significant decision guidance for the safe operation and planning of power systems.

So far, the existing node importance evaluation methods, including degree and betweenness measure, closeness and PageRank (PR) method based on complex network theory and social network analysis, have been reported in [4]-[14]. In the graph theory, the degree of a node is defined as the number of transmission lines adjacent to this node, and a node with the larger value of degree tends to have higher influence in the power network [8]. The betweenness expresses the dominance level of a node from the viewpoint of the whole power network [9]. Vertex-betweenness of a given node is the ratio of the number of shortest paths passing through the node over the number of all the shortest paths between vertex pairs. An electrical betweenness (EBT) method combining the capacity and distribution of generators and loads was proposed in [10] to evaluate and rank the critical nodes in power grids. In the complex network, the node closeness centrality reveals the compactness level from one node to other nodes, and also indicates the center extent and indirect influence of the node to other nodes [8]. The larger value of node closeness denotes the centrality and significance of the node to other nodes. With a better definition of electrical distance to express the system vulnerability, the closeness method has been adopted in [11] to identify important nodes or branches in the power system. Besides, a multi-criterion measuring method with adjustable parameters is proposed in [12], considering the node degree, star degree and betweenness, to estimate the importance of network nodes.

Furthermore, the PR is proposed in [13] to work by counting the number and quality of links to a given node in order to determine an estimation of the node significance, and a node evaluation method based on PR was proposed in [14] considering the importance of nodal load, nodal load capacity and network topology. However, the PR based method in [14] only considers the load loss of a single node when suffering the node attacks, and the loss of loads resulted from the cascading failure process is not involved. In 2011, Lv et al. [15] proposed a variant of PR, called LeaderRank (LR), in which a ground 
node is introduced to connect with all other nodes by a bidirectional edge to the original network. It has been demonstrated in [16] that LR outperforms PR with faster convergence and ranking effectiveness, wider applicability to any type of network, as well as higher robustness against the noisy data and initial conditions. In the recent years, modern power grids become more and more vulnerable with the increasing grid-connected renewable energy generations (REGs) [17],[18]. Because of the intermittent and volatile nature of wind and solar energy sources, the power flow distribution when integrating large amounts of intermittent REGs could differ considerably from the power system with traditional generation sources [19], and the uncertainties in the power flow through transmission lines would further contribute to extend the failure size-scale when the cascading failure occurs. Therefore, the problem of node importance evaluation of power grids becomes even more complicated with the intermittent and volatile REGs.

In this paper, an electrical LeaderRank (ELR) method is proposed to assess and identify the important nodes of power grids considering the uncertainties of renewable energy. Due to the influence of variable wind and solar energy sources on the power system vulnerability, the output uncertainties of REGs are modelled as interval numbers to investigate the interval uncertain transmission power, and a cascading failure simulation model is formulated based on the interval DC optimal power flow (DC-OPF) to measure the loss of loads after the power system suffering from attacks. Consequently, the interval power flow distribution and load loss caused by cascading failures are taken into consideration to propose an interval weighted strategy of value functions for the ELR method in order to enhance the identification accuracy of system important nodes. Comparative simulations of the proposed ELR with other node evaluation methods have been fully tested and benchmarked on a modified IEEE 118-bus power system and the Western Liaoning Power Grid of China, and comparative results on four performance measures, including average load loss, maximum amount of load, network transmission efficiency, and system connectivity index, have demonstrated the validity and superiority of the proposed approach.

\section{Modelling of Cascading Failure with REGs}

\subsection{Interval DC-OPF with REGs}

The volatility and intermittency always accompany the wind and solar energy sources due to weather variability. For example, wind power generation is generally influenced by the fluctuating speed and orientation of wind [20]. The uncertainties of these REGs would lead to uncertainties in the power flow distribution of power grids, and may cause reliability issues especially for the systems with high renewable energy penetration [19]. Here, the uncertainties in wind speed, illumination intensity and solar cell tem- 
perature can be formulated as the interval numbers to model the wind and solar generation outputs so as to formulate the interval DC-OPF problem [21]. With the implementation of interval DC-OPF, the interval uncertainties in the transmission power of each line can be obtained and indicated by interval numbers. Firstly, the definition of interval wind speed is denoted as,

$$
w^{\mathrm{I}}=\left[w^{\mathrm{L}}, w^{\mathrm{U}}\right]=\left\{w \mid w^{\mathrm{L}} \leq w \leq w^{\mathrm{U}}\right\}
$$

where $w^{\mathrm{I}}$ is an interval variable to represent the uncertainty of wind speed; $w^{\mathrm{L}}$ and $w^{\mathrm{U}}$ are the lower and upper bounds of wind speed interval. Based on the elementary operations of interval arithmetic [22], the linearized output power curves of wind turbine generation (WTG) in [18],[20] can be transformed into the interval model [23],

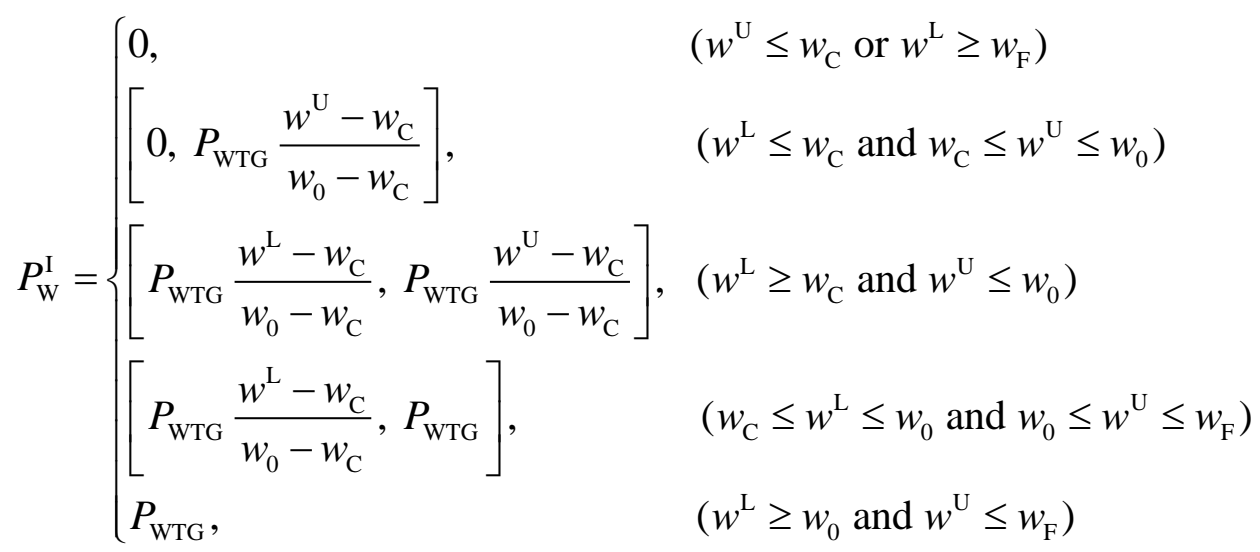

where $P_{\mathrm{W}}^{\mathrm{I}}$ represents the interval WTG output; $P_{\mathrm{WTG}}$ is the rated power capacity of the WTG; $w_{0}, w_{\mathrm{C}}$, and $w_{\mathrm{F}}$ are the rated wind speed, cut-in wind speed and cut-off wind speed of wind turbine, respectively. Also, the interval output power of a photovoltaic (PV) system can be expressed as follows [23],

$$
H_{\mathrm{ING}}^{\mathrm{I}}=\left[H_{\mathrm{ING}}^{\mathrm{L}}, H_{\mathrm{ING}}^{\mathrm{U}}\right], \quad T_{\mathrm{C}}^{\mathrm{I}}=\left[T_{\mathrm{C}}^{\mathrm{L}}, T_{\mathrm{C}}^{\mathrm{U}}\right]
$$

$$
P_{\mathrm{P}}^{\mathrm{I}}=\left[\frac{P_{\mathrm{PV}}}{H_{\mathrm{STC}}}\left(H_{\mathrm{ING}}^{\mathrm{L}}+\varepsilon_{\mathrm{T}} H_{\mathrm{ING}}^{\mathrm{L}} T_{\mathrm{C}}^{\mathrm{L}}-\varepsilon_{\mathrm{T}} H_{\mathrm{ING}}^{\mathrm{U}} T_{0}\right), \frac{P_{\mathrm{PV}}}{H_{\mathrm{STC}}}\left(H_{\mathrm{ING}}^{\mathrm{U}}+\varepsilon_{\mathrm{T}} H_{\mathrm{ING}}^{\mathrm{U}} T_{\mathrm{C}}^{\mathrm{U}}-\varepsilon_{\mathrm{T}} H_{\mathrm{ING}}^{\mathrm{L}} T_{0}\right)\right]
$$

where $P_{\mathrm{P}}^{\mathrm{I}}$ is the interval generation output of $\mathrm{PV}$ array; $P_{\mathrm{PV}}$ denotes the rated power capacity of $\mathrm{PV}$ array; $H_{\mathrm{ING}}^{\mathrm{I}}$ and $H_{\mathrm{STC}}$ denote the interval of incident irradiance and the irradiance at standard test condition 1000 $\mathrm{W} / \mathrm{m}^{2}$, respectively; $\alpha_{\mathrm{T}}$ is the temperature coefficient of power; $T_{0}$ is the reference rated temperature; $T_{\mathrm{C}}^{\mathrm{I}}$ is the interval of PV temperature; $H_{\mathrm{ING}}^{\mathrm{L}}$ and $H_{\mathrm{ING}}^{\mathrm{U}}, T_{\mathrm{C}}^{\mathrm{L}}$ and $T_{\mathrm{C}}^{\mathrm{U}}$ are the lower and upper bounds of the irradiance and cell temperature interval, respectively.

In order to analyze the cascading failure process and node importance considering the uncertainties of renewable energy sources, the interval DC-OPF model in [24],[25] is adopted in this study to calculate the interval power flow in each transmission line derived from the interval uncertainties of wind power $P_{\mathrm{W}}^{\mathrm{I}}$ and solar power $P_{\mathrm{P}}^{\mathrm{I}}$. In the DC-OPF model, the adjustments on the outputs of generators and load shedding can be implemented to eliminate the transmission line or transformer overloads, and the optimi- 
zation objective is to minimize the total generation cost [8], as follows,

$$
\begin{array}{ll}
\min & F=\sum_{i \in S_{\mathrm{G}}} c_{i} P_{\mathrm{G} i} \\
\text { s.t. } & \boldsymbol{P}^{\mathrm{PF}}=\boldsymbol{A} \boldsymbol{P}^{\mathrm{SP}} \\
& \sum_{i \in S_{\mathrm{G}}} P_{\mathrm{G} i}-\sum_{j \in S_{\mathrm{D}}} P_{\mathrm{D} j}=0 \\
& P_{\mathrm{G} i, \text { min }} \leq P_{\mathrm{G} i} \leq P_{\mathrm{G} i, \text { max }}, \quad i \in S_{\mathrm{G}} \\
& -P_{i j, \text { max }} \leq P_{i j} \leq P_{i j, \max }, \quad i, j \in S_{\mathrm{B}} \\
& P_{\mathrm{W}}^{\mathrm{I}}=\left[P_{\mathrm{W}}^{\mathrm{L}}, P_{\mathrm{W}}^{\mathrm{U}}\right], \quad P_{\mathrm{P}}^{\mathrm{I}}=\left[P_{\mathrm{P}}^{\mathrm{L}}, P_{\mathrm{P}}^{\mathrm{U}}\right]
\end{array}
$$

where $c_{i}$ are the generation cost coefficients for the $i$ th generator; $P_{\mathrm{G} i}$ and $P_{\mathrm{D} j}$ are the active power outputs of generator at node $i$ and load at node $j$, respectively; $\boldsymbol{P}^{\mathrm{PF}}, \boldsymbol{A}$ and $\boldsymbol{P}^{\mathrm{SP}}$ denote the matrix of branch power flow, branch admittance matrix, and the matrix of nodal power injection, respectively; $P_{i j}$ is the power flow in transmission lines; $S_{\mathrm{G}}, S_{\mathrm{D}}$ and $S_{\mathrm{B}}$ represent the sets of generator nodes, load nodes and power system buses, respectively. The problem constraints include the DC power flow equation, power balance equation, load shedding limits, generator output limits and transmission capacity limits [26].

Based on the direct interval matching and range arithmetic principles of extreme value intervals in [21],[27], the minimization of interval DC-OPF function $F^{\mathrm{I}}(\boldsymbol{X})=\left[F^{\mathrm{L}}(\boldsymbol{X}), F^{\mathrm{U}}(\boldsymbol{X})\right]$ with the optimal interval $\boldsymbol{X}^{*}$ can be transformed into two deterministic linear programming problems to minimize the upper and lower bounds of DC-OPF objective functions [25], as follow,

$$
\begin{array}{r}
F^{\mathrm{I}}\left(\boldsymbol{X}^{*}\right)=\left[F^{\mathrm{L}}\left(\boldsymbol{X}^{*}\right), F^{\mathrm{U}}\left(\boldsymbol{X}^{*}\right)\right]=\min \left(F^{\mathrm{I}}(\boldsymbol{X})=\left[F^{\mathrm{L}}(\boldsymbol{X}), F^{\mathrm{U}}(\boldsymbol{X})\right]\right) \\
\Leftrightarrow\left\{\begin{array}{l}
F^{\mathrm{L}}\left(\boldsymbol{X}^{*}\right)=\min F^{\mathrm{L}}(\boldsymbol{X}) \\
F^{\mathrm{U}}\left(\boldsymbol{X}^{*}\right)=\min F^{\mathrm{U}}(\boldsymbol{X})
\end{array}\right. \\
\text { s.t. } \boldsymbol{X}^{\mathrm{L}} \leq \boldsymbol{X} \leq \boldsymbol{X}^{\mathrm{U}}
\end{array}
$$

where $F^{\mathrm{I}}(\boldsymbol{X})$ denotes the interval objective function with lower bound $F^{\mathrm{L}}(\boldsymbol{X})$ and upper bound $F^{\mathrm{U}}(\boldsymbol{X})$ in the interval DC-OPF model; $\boldsymbol{X}$ is the decision variable vector to be optimized in DC-OPF model (4); $\boldsymbol{X}^{\mathrm{L}}$ and $\boldsymbol{X}^{\mathrm{U}}$ are the lower and upper bound vectors of OPF decision variables, respectively. Here, the interior point method is used to solve the two deterministic linear programming problems [25], and the lower and upper bounds of interval solution can then be obtained with the interval DC-OPF implementation. With the interval DC-OPF results, the interval power flow, $P_{i j}^{\mathrm{I}}$, in each transmission line can be obtained, and the adjacency matrix $\boldsymbol{V}$ can be employed to indicate the directed topological structure of power grids [8]. Here, the element $v_{i j}$ in matrix $\boldsymbol{V}$ represents the power direction of adjacent node pairs for the directed topology graph. Due to the uncertainties of interval power flow, the lower and upper boundary adjacency matrices, $\boldsymbol{V}^{\mathrm{L}}$ and $\boldsymbol{V}^{\mathrm{U}}$, can be formulated based on the interval power flow $P_{i j}^{\mathrm{L}}$ and $P_{i j}^{\mathrm{U}}$ in the transmission 
$1 \quad$ line between nodes $i$ and $j$. If the power flow $P_{i j}^{\mathrm{L}}$ is transmitted from node $i$ to node $j$, the element $v_{i j}^{\mathrm{L}}$ in

2 matrix $V^{\mathrm{L}}$ is set to 1; otherwise, $v_{i j}^{\mathrm{L}}$ is set to 0 . Similarly, the element $v_{i j}^{\mathrm{U}}$ in matrix $\boldsymbol{V}^{\mathrm{U}}$ can be assigned 3 based on the upper boundary value of transmission power flow $P_{i j}^{\mathrm{U}}$.

\subsection{Interval DC-OPF based cascading failure model}

The power grid is a complex electricity network with multiple stochastic uncertainty sources. The previous studies found that power system can be equivalent to a complex network, and it is discovered the small-world network characteristics of western United States power grid in [28]. In order to analyze the nodal vulnerability of power grids with the uncertainties from REGs, the power grid can be formulated to a topological model with each bus regarded as a node [7]. Based on the self-organized criticality [29], a complex system would be in danger if it comes to a critical state. In a critical state with large probability of major interruptions, even a tiny disturbance could lead to an uncontrollable system collapse. The sand pile model in [8] can be used to explain the process of power grid cascading failure with system dynamics. With the interval DC-OPF model, the power flow distribution continuously varies with load growth. Once the load increases up to a certain level, the power system may evolve into a critical state, and the line outage triggered by the overload or some malfunction may cause the cascading failure [30],[31].

In modern electricity transmission systems, the electricity devices employed are often highly stressed due to the economic compromise and heavy load demand, and hence the power system may close to the critical state with the integration of high penetration of variable REGs. Once the malfunction or load growth makes a specific component fail to transmit power, the system power flow would be redistributed, and the critical state may be resulted. Consequently, there would be an increase in the loading of other system components, which might cause another round of fail and overloading, i.e. the propagation of failures [32]. As power blackouts would lead to significant social and economic consequences, it is important to model the complex dynamics of cascading failures of power grids and implement the preventive or corrective measures in mitigating the risks of large-scale blackouts [33]. The ORNL-PSerc-Alaska (OPA) model is a blackout model proposed in [34] to simulate the operating behaviors of power systems based on the sand pile model with self-organized characteristics, and the global dynamic characteristics of power system cascading failures can be investigated through the DC-OPF calculation with the varying load demand, power generation and transmission line capacities.

In this paper, an interval DC-OPF based cascading failure model considering the uncertainties of renewable energy sources is formed based on an improved OPA model in [34], as shown in Fig. 1. Cascading failures can be simulated by cutting off those heavily loaded transmission lines with a certain proba- 
1 bility and the load loss can represent the scale of the failure. The whole procedures of the proposed model 2 can be described in the following flowchart:

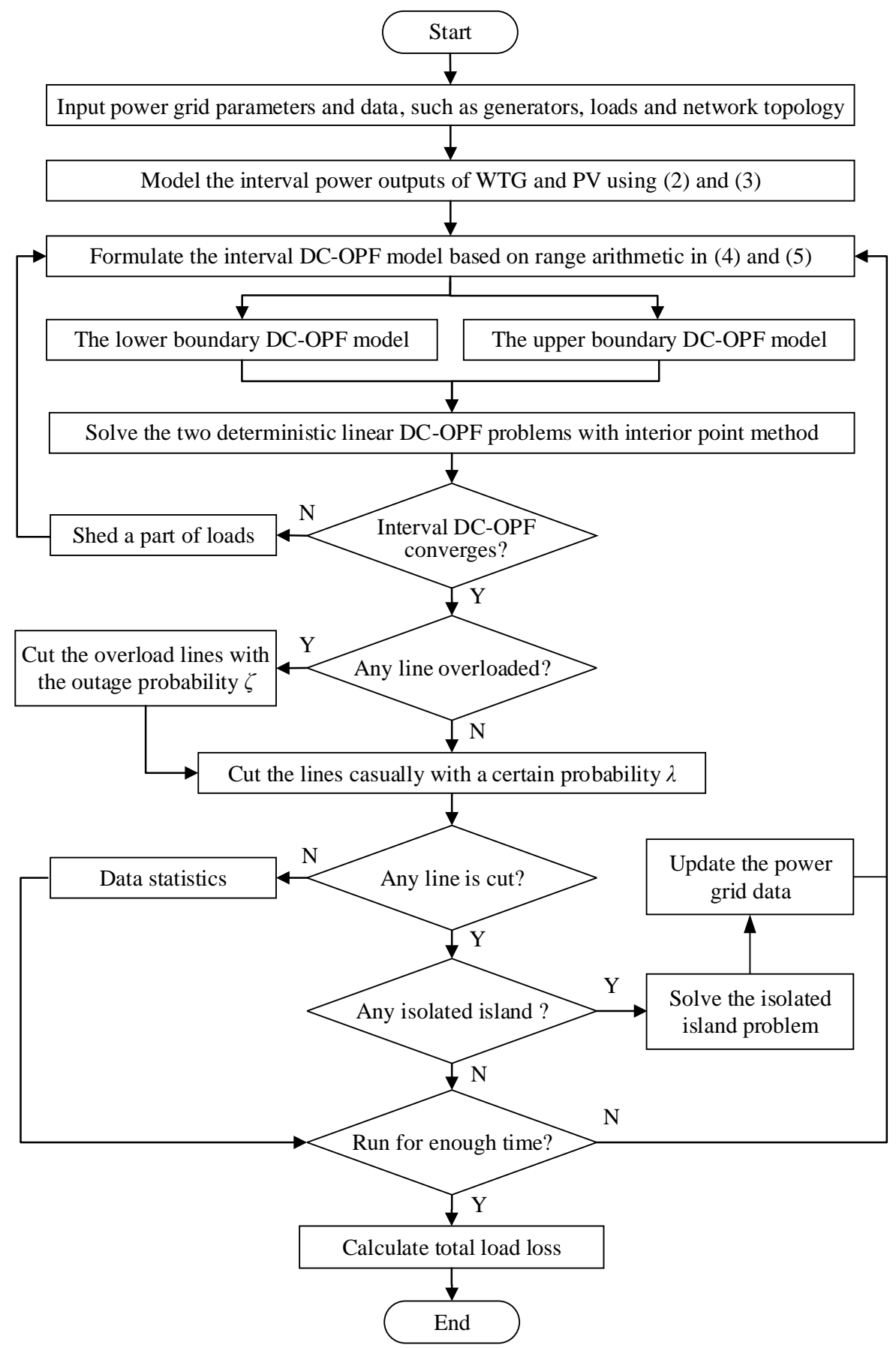

Fig. 1 Flowchart of interval DC-OPF based cascading failure model with REGs

Step 1: Input the power grid parameters, including the load parameters, generator outputs, and sys6 tem topology and transmission lines, and so on.

Step 2: Model the interval renewable generations. The interval outputs of WTG and PV can be formulated from uncertain wind speed and illumination intensity intervals using (2) and (3), respectively. 
Step 3: Formulate the interval DC-OPF model in (4), and then the model can be transformed into two deterministic optimization problems, namely upper and lower boundary DC-OPF problems [21], based on interval analysis and range arithmetic principles in (5).

Step 4: Solve the two linear programming problems of lower and upper boundary DC-OPF models using the interior point method.

Step 5: If both of the lower and upper boundary optimizations converge, go to Step 4; otherwise, incrementally shed loads until the OPF converges, then go to Step 4.

Step 6: Remove the overloaded lines. Based on the obtained interval power flow in each transmission line, if the ratio of the average of interval transmission power to its capacity is greater than a threshold $\sigma$, this transmission line is regarded to be overloaded and may be eliminated with a probability $\zeta$. In this study, the line overload threshold $\sigma$ and outage probability $\zeta$ are set to 0.7 and 0.3 [8], respectively.

Step 7: Eliminate lines randomly with a certain probability $\lambda$, which simulates the system fault that is not caused by line overloading, then go to Step 6. The outage probability is determined by the ratio of the average number of failures on the transmission lines in the power system, and the outage probability can be set to 0.001 based on the historical outage data in China in 2014 [8].

Step 8: If any line has been eliminated, update power grid parameters and go to Step 7; otherwise, go to Step 8 .

Step 9: With the new power grid topological data, if any isolated island is detected, computing the load and generation capacity of each island with the method in [34], then update the power grid data and go to Step 3; otherwise, go to Step 8.

Step 10: Record the number of runs from Step 3 to Step 7. If the runs are implemented with enough time, go to Step 9; otherwise, go to Step 3. In this study, the maximum iterative runs are set to 10000 [8].

Step 11: Finally, calculate the total load loss in the cascading failure process.

\section{Electrical LeaderRank Method}

LeaderRank (LR) is an adaptive and parameter-free ranking algorithm to identify the influential node in the complex networks [15]. It has been demonstrated in [16],[35] that the performance of the LR exhibits the ranking effectiveness, fast convergence and high robustness. For a directed network with $N$ nodes and $M$ directed links, a special node, called ground node, which connects all others by bidirectional links, is added to facilitate the node rankings. Hence, the new network is strongly connected with $N+1$ nodes and $M+2 N$ links. In the LR evaluation, the $L R$ values, which indicate the importance degree of nodes, can be iteratively assigned to each node. Firstly, the initial $L R$ value of ground node is set to 0 and 
the initial $L R$ values of other nodes are set to 1 . Then, the $L R$ value of each node can be obtained from its in-linked nodes which evenly allocate their $L R$ values to the neighbours through the directed links, and the nodes obtained the $L R$ values are called out-linked nodes of the in-linked node. Finally, the value of the ground node is uniformly distributed to all other nodes to conserve values on the nodes of interest [15]. The $L R$ value function of node $i$ is formulated as follows,

$$
L R_{i}=\sum_{j=1}^{N+1} \frac{v_{j i}}{k_{j-\text { out }}} L R_{j}+\frac{L R_{g}}{N}
$$

where $N$ is the number of nodes except for the ground node in the network; $L R_{j}$ represents the $L R$ value of node $j$, and $L R_{g}$ is the $L R$ values of ground node at final state when the $L R$ value of each node converge to a unique steady state; $k_{j-\text { out }}$ is the number of out-linked nodes with node $j ; v_{j i}$ can be obtained by the direct adjacent matrix $\boldsymbol{V}$.

In this paper, the ELR method is further proposed to evaluate the node importance for power systems with REGs, and the higher ELR value represents the higher importance of the grid node. For the complex power gird with renewable energy uncertainties, the uniformly distribution strategy of $L R$ value in (6) is not applicable due to different power flow through different transmission lines and different loads among the system nodes. Also, the nodes with a number of in-linked and out-linked nodes tend to more important than others. Therefore, a novel weighted proportional strategy of value functions is proposed to distribute the ELR values of power grid nodes and ground node to their out-linked grid nodes, considering the system topological structure, transmission power flow and the loss of loads caused by cascading failures. Furthermore, due to the interval power flow distribution as well as the corresponding adjacency matrices resulted from the interval DC-OPF model, the ELR values obtained from the proposed evaluation method should also be interval numbers. Based on the weighted mechanism of value function in the LR method [35], the weighted importance degrees are allowed and assigned to the nodes with different in-linked and out-linked nodes in this study. For the node $i$, the interval values of $E L R, E L R_{i}^{\mathrm{L}}$ and $E L R_{i}^{\mathrm{U}}$, can be derived from the lower and upper boundary adjacency matrices $\boldsymbol{V}^{\mathrm{L}}$ and $\boldsymbol{V}^{\mathrm{U}}$, as follows,

$$
E L R_{i}^{\mathrm{I}}=\left[E L R_{i}^{\mathrm{L}}, E L R_{i}^{\mathrm{U}}\right] \Leftrightarrow\left\{\begin{array}{l}
E L R_{i}^{\mathrm{L}}=\sum_{j=1}^{N+1} \alpha_{(j \rightarrow i)}^{\mathrm{L}} \cdot \frac{v_{i j}^{\mathrm{L}}}{k_{j \text {-out }}^{\mathrm{L}}} \cdot E L R_{j}^{\mathrm{L}}+E L R_{g}^{\mathrm{L}} \cdot \beta_{(g \rightarrow i)}^{\mathrm{L}} \\
E L R_{i}^{\mathrm{U}}=\sum_{j=1}^{N+1} \alpha_{(j \rightarrow i)}^{\mathrm{U}} \cdot \frac{v_{i j}^{\mathrm{U}}}{k_{j \text {-out }}^{\mathrm{U}}} \cdot E L R_{j}^{\mathrm{U}}+E L R_{g}^{\mathrm{U}} \cdot \beta_{(g \rightarrow i)}^{\mathrm{U}}
\end{array}\right.
$$

where $\alpha_{(j \rightarrow i)}^{\mathrm{I}}=\left[\alpha_{(j \rightarrow i)}^{\mathrm{L}}, \alpha_{(j \rightarrow i)}^{\mathrm{U}}\right]$ represents the distribution weight of $E L R$ values from node $j$ to node $i$; $E L R_{j}^{\mathrm{I}}=\left[E L R_{j}^{\mathrm{L}}, E L R_{j}^{\mathrm{U}}\right]$ represents the interval $E L R$ value of node $j ; k_{j \text {-out }}=\left[k_{j \text {-out }}^{\mathrm{L}}, k_{j \text {-out }}^{\mathrm{U}}\right]$ represents the number of out-linked nodes connected by node $j$, and the elements $v_{i j}^{\mathrm{L}}$ and $v_{i j}^{\mathrm{U}}$ can be obtained from the 
1 direct adjacent matrices $V^{\mathrm{L}}$ and $\boldsymbol{V}^{\mathrm{U}}$, respectively; $E L R_{g}^{\mathrm{I}}=\left[E L R_{g}^{\mathrm{L}}, E L R_{g}^{\mathrm{U}}\right]$ denotes the interval $E L R$ value of 2 the ground node, and $\beta_{(g \rightarrow i)}^{\mathrm{I}}=\left[\beta_{(g \rightarrow i)}^{\mathrm{L}}, \beta_{(g \rightarrow i)}^{\mathrm{U}}\right]$ denotes the distribution weight of $E L R$ values from ground node to node $i$.

In the proposed ELR method, the determination on the distribution weight from nodes $j$ to $i, \alpha_{(j \rightarrow i)}$ should involve the system topological structure and interval transmission power flow, and its weighted rules can be formulated based on interval power flow distributions, as follows,

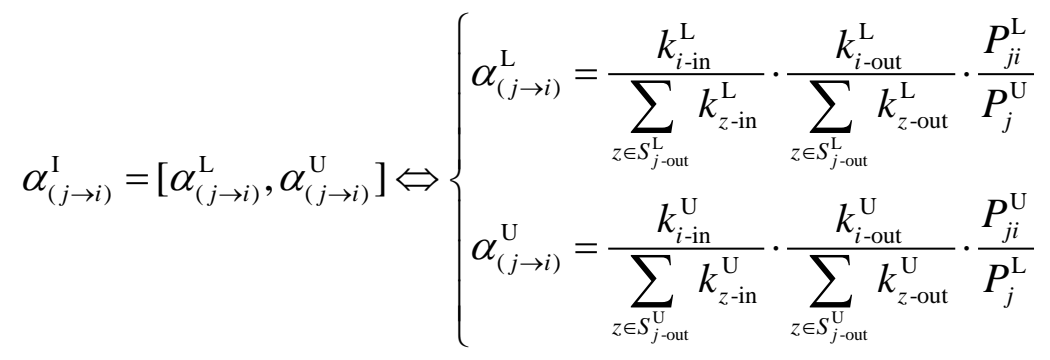

$$
\begin{aligned}
& \frac{P_{j i}^{\mathrm{L}}}{P_{j}^{\mathrm{U}}} \text { or } \frac{P_{j i}^{\mathrm{U}}}{P_{j}^{\mathrm{L}}}= \begin{cases}\frac{P_{j i}^{\mathrm{L}}}{P_{j}^{\mathrm{U}}} \text { or } \frac{P_{j i}^{\mathrm{U}}}{P_{j}^{\mathrm{L}}}, & j \in \Omega_{1} \\
1, & j \in \Omega_{2} \\
\frac{1}{N}, & j \text { is the ground node }\end{cases}
\end{aligned}
$$

where $k_{i \text {-in }}=\left[k_{i \text {-in }}^{\mathrm{L}}, k_{i \text {-in }}^{\mathrm{U}}\right]$ and $k_{i \text {-out }}=\left[k_{i \text {-out }}^{\mathrm{L}}, k_{i \text {-out }}^{\mathrm{U}}\right]$ represent the numbers of in-linked nodes and out-linked nodes of node $i$, respectively; $S_{j \text {-out }}=\left[S_{j \text {-out }}^{\mathrm{L}}, S_{j \text {-out }}^{\mathrm{U}}\right]$ denotes the lower and upper boundary sets of outlinked nodes of node $j$, and $z$ denotes the node from set $S_{j \text {-out }}, k_{z \text {-in }}=\left[k_{z \text {-in }}^{\mathrm{L}}, k_{z \text {-in }}^{\mathrm{U}}\right]$ and $k_{z \text {-out }}=\left[k_{z \text {-out }}^{\mathrm{L}}, k_{z \text {-out }}^{\mathrm{U}}\right]$ are the numbers of in-linked nodes and out-linked nodes of node $z$ under the interval power flow; $P_{j}^{\mathrm{I}}=\left[P_{j}^{\mathrm{L}}, P_{j}^{\mathrm{U}}\right]$ indicates the total amount of power transfer from node $j$ to its out-linked nodes with the interval transmission power flow; $\Omega_{1}$ is the node set which has at least one out-linked node except for the ground node; $\Omega_{2}$ is the node set which only has the ground node as its out-linked node.

The weight factor $\beta_{(g \rightarrow i)}$ in (7) should take into account the system topological structure and load loss due to cascading failure scenarios. If the failure of a node would cause a large amount of load loss in the power system, the larger ELR value should be assigned from the ground node to this node so as to indicate its higher importance degree. Hence, the weight $\beta_{(g \rightarrow i)}$ can be determined as follows,

$$
\beta_{(g \rightarrow i)}^{\mathrm{I}}=\left[\beta_{(g \rightarrow i)}^{\mathrm{L}}, \beta_{(g \rightarrow i)}^{\mathrm{U}}\right] \Leftrightarrow\left\{\begin{array}{l}
\beta_{(g \rightarrow i)}^{\mathrm{L}}=\frac{k_{i \text {-out }}^{\mathrm{L}}+k_{i \text {-in }}^{\mathrm{L}}}{\sum_{y \in S_{\mathrm{B}}}\left(k_{y-\text { out }}^{\mathrm{L}}+k_{y \text {-in }}^{\mathrm{L}}\right)} \cdot \frac{P_{\mathrm{LL} i}}{\sum_{y \in S_{\mathrm{B}}} P_{\mathrm{LL} y}} \\
\beta_{(g \rightarrow i)}^{\mathrm{U}}=\frac{k_{i \text {-out }}^{\mathrm{U}}+k_{i \text {-in }}^{\mathrm{U}}}{\sum_{y \in S_{\mathrm{B}}}\left(k_{y \text {-out }}^{\mathrm{U}}+k_{y \text {-in }}^{\mathrm{U}}\right)} \cdot \frac{P_{\mathrm{LL} i}}{\sum_{y \in S_{\mathrm{B}}} P_{\mathrm{LL} y}}
\end{array}\right.
$$

1 where $S_{\mathrm{B}}$ represents the set of power grid nodes expect for the ground node, and $y$ denotes the node from set $S_{\mathrm{B}} ; P_{\mathrm{LL} i}$ is the load loss from cascading failure scenarios when node $i$ is attacked, which can be simu- 
lated and obtained with the interval DC-OPF based cascading failure model in Fig. 1.

In summary, the node importance ranking of power grids with REGs can be achieved with (7)-(10), and the flowchart to implement the proposed ELR method is illustrated in Fig. 2. The interval ELR value of each node can be obtained with the iterative ELR value function in (7). If the variation between two 5 iterative ELR values of each node is small enough as the difference value is less than 0.001 , the interval $6 E L R$ value is regarded to be convergent. It should be pointed out that the ground node, as an additional node, forms virtual connections with all existing power grid nodes via bidirectional edges. After the con8 vergence of lower and upper boundary ELR values of all the nodes, the value of ground node will be al9 located to all the grid nodes based on the weighted rules in (10). As a result, the node importance of power grids can be evaluated and sorted according to the interval number comparisons on nodal ELR values 11 [22], and the higher ELR value of a node indicates the higher node importance in the power system.

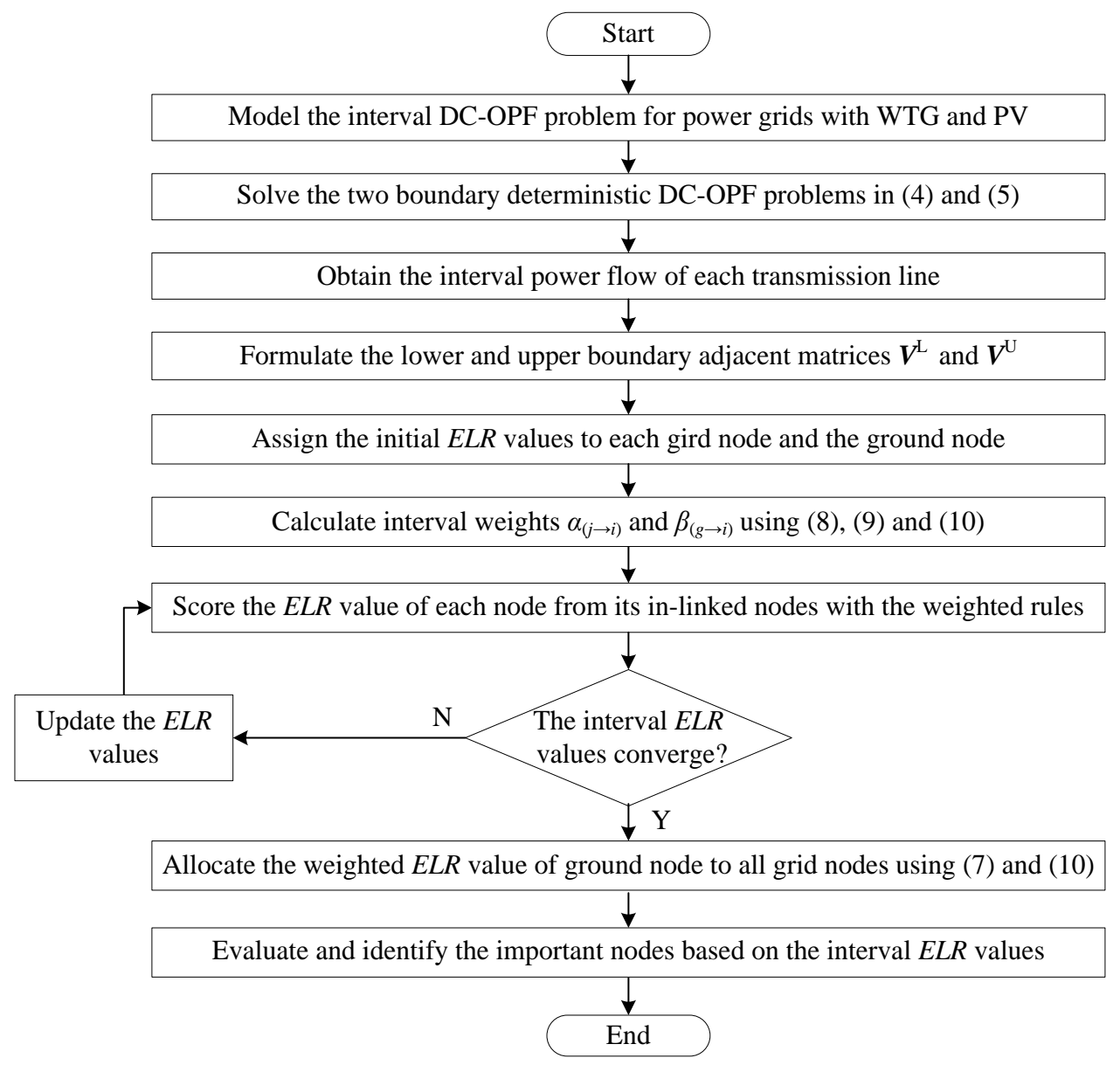

Fig. 2 Flowchart of ELR method for node importance evaluation of power grids 
In this paper, a modified IEEE 118-bus power system, as shown in Fig. 3, is adopted to demonstrate the performance of the proposed ELR method for node importance evaluation on various system vulnerability metrics, and the detailed power grid topology and data are available in [36]. Four wind farms on bus 25, 66, 74 and 104 and a PV power station on bus 10 are set for grid-integration in the 118-bus power

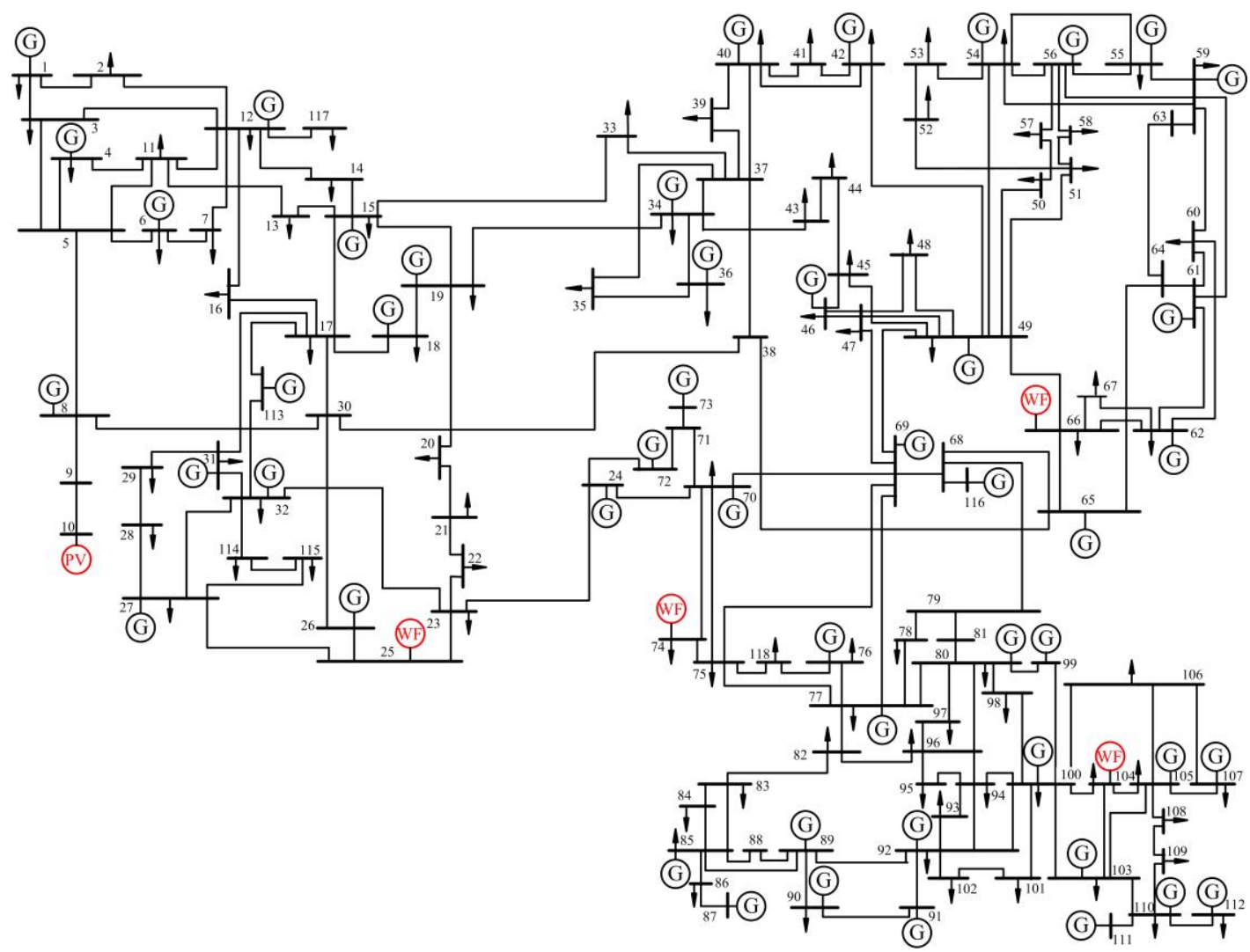

Fig. 3 IEEE 118-bus power system with wind farms and PV power stations 


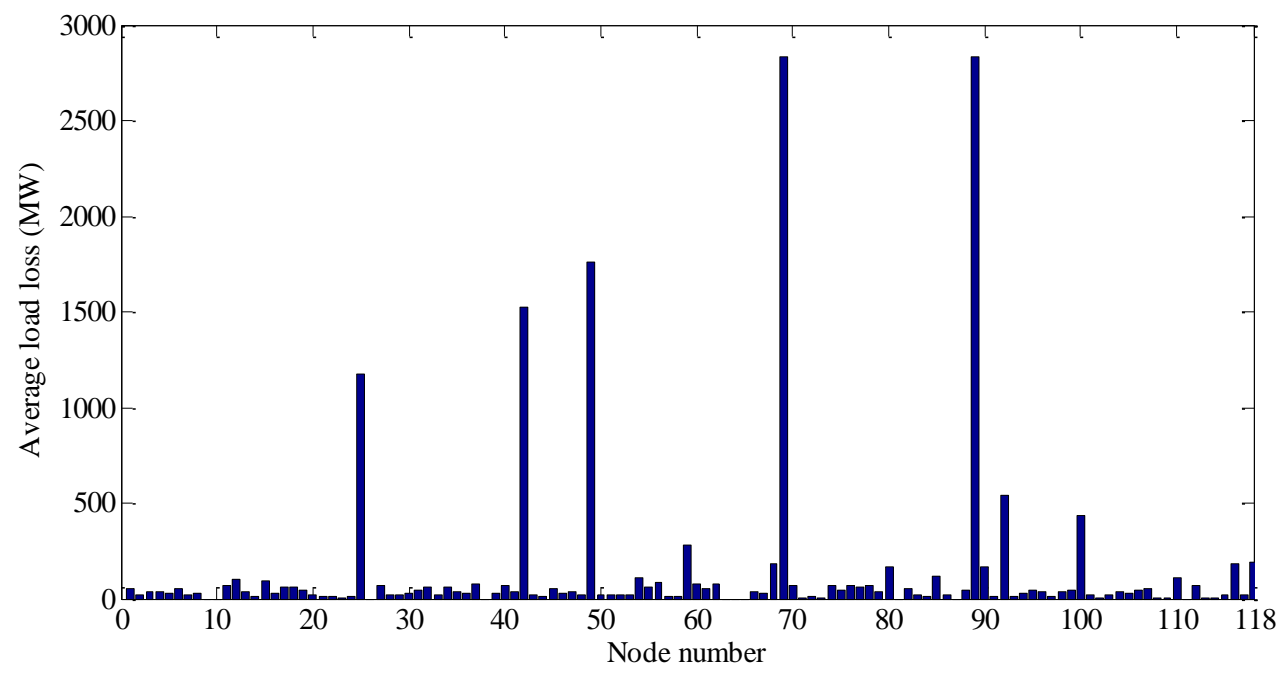

Fig. 4 Average load loss of 118-bus power system after each node attack

Table 1 The ELR intervals of 118-bus power system for node importance evaluation

\begin{tabular}{|c|c|c|c|c|c|c|c|c|}
\hline Node No. & $E L R^{\mathrm{L}}$ value & $E L R^{\mathrm{U}}$ value & Node No. & $E L R^{\mathrm{L}}$ value & $E L R^{\mathrm{U}}$ value & Node No. & $E L R^{\mathrm{L}}$ value & $E L R^{\mathrm{U}}$ value \\
\hline 1 & 0.085 & 1.549 & 41 & 0.687 & 0.917 & 81 & 0.377 & 0.813 \\
\hline 2 & 0.338 & 0.401 & 42 & 0.070 & 1.074 & 82 & 0.264 & 1.256 \\
\hline 3 & 0.232 & 0.857 & 43 & 0.140 & 0.808 & 83 & 0.070 & 0.789 \\
\hline 4 & 0.085 & 1.618 & 44 & 0.811 & 0.987 & 84 & 0.269 & 0.572 \\
\hline 5 & 1.541 & 1.873 & 45 & 0.810 & 0.811 & 85 & 1.371 & 1.414 \\
\hline 6 & 0.705 & 0.809 & 46 & 0.634 & 1.123 & 86 & 0.116 & 0.808 \\
\hline 7 & 0.608 & 0.812 & 47 & 0.671 & 0.758 & 87 & 0.070 & 0.812 \\
\hline 8 & 0.085 & 1.841 & 48 & 0.502 & 0.708 & 88 & 0.636 & 0.770 \\
\hline 9 & 0.808 & 0.915 & 49 & 2.892 & 2.938 & 89 & 3.221 & 3.277 \\
\hline 10 & 0.070 & 0.811 & 50 & 0.275 & 0.780 & 90 & 0.085 & 0.812 \\
\hline 11 & 0.646 & 1.746 & 51 & 0.085 & 1.514 & 91 & 0.234 & 0.313 \\
\hline 12 & 2.287 & 2.790 & 52 & 0.796 & 0.809 & 92 & 2.624 & 3.480 \\
\hline 13 & 0.302 & 0.563 & 53 & 0.660 & 0.811 & 93 & 0.387 & 0.671 \\
\hline 14 & 0.689 & 0.704 & 54 & 0.811 & 2.044 & 94 & 0.085 & 2.289 \\
\hline 15 & 1.374 & 2.182 & 55 & 0.274 & 0.614 & 95 & 0.535 & 0.648 \\
\hline 16 & 0.199 & 0.621 & 56 & 0.867 & 2.345 & 96 & 0.232 & 1.575 \\
\hline 17 & 1.961 & 2.081 & 57 & 0.070 & 1.405 & 97 & 0.070 & 0.965 \\
\hline 18 & 0.511 & 0.569 & 58 & 0.070 & 1.192 & 98 & 0.085 & 0.361 \\
\hline 19 & 1.420 & 1.548 & 59 & 0.183 & 0.763 & 99 & 0.085 & 0.378 \\
\hline 20 & 0.808 & 0.881 & 60 & 0.610 & 1.206 & 100 & 2.054 & 3.418 \\
\hline 21 & 0.810 & 0.812 & 61 & 1.062 & 1.926 & 101 & 0.127 & 0.758 \\
\hline 22 & 0.811 & 0.812 & 62 & 0.309 & 1.005 & 102 & 0.810 & 0.815 \\
\hline 23 & 0.811 & 1.490 & 63 & 0.085 & 2.238 & 103 & 1.163 & 1.549 \\
\hline 24 & 0.678 & 0.897 & 64 & 1.144 & 1.290 & 104 & 0.085 & 0.816 \\
\hline 25 & 0.810 & 1.785 & 65 & 1.312 & 1.909 & 105 & 1.177 & 1.888 \\
\hline 26 & 0.085 & 1.515 & 66 & 1.339 & 1.967 & 106 & 0.606 & 1.301 \\
\hline 27 & 0.812 & 1.545 & 67 & 0.070 & 1.412 & 107 & 0.070 & 0.085 \\
\hline 28 & 0.508 & 0.808 & 68 & 0.777 & 1.550 & 108 & 0.360 & 0.808 \\
\hline 29 & 0.812 & 0.978 & 69 & 2.762 & 4.053 & 109 & 0.529 & 0.811 \\
\hline 30 & 0.408 & 1.913 & 70 & 0.404 & 1.831 & 110 & 1.536 & 1.546 \\
\hline 31 & 0.437 & 0.972 & 71 & 1.081 & 1.261 & 111 & 0.070 & 0.782 \\
\hline 32 & 0.963 & 1.482 & 72 & 0.070 & 1.130 & 112 & 0.070 & 0.085 \\
\hline 33 & 0.149 & 0.257 & 73 & 0.070 & 0.899 & 113 & 0.070 & 1.193 \\
\hline 34 & 0.382 & 2.229 & 74 & 0.098 & 0.323 & 114 & 0.942 & 1.031 \\
\hline 35 & 0.085 & 1.060 & 75 & 0.830 & 1.609 & 115 & 0.070 & 1.330 \\
\hline 36 & 0.070 & 1.314 & 76 & 0.085 & 0.417 & 116 & 0.070 & 1.047 \\
\hline 37 & 1.171 & 2.470 & 77 & 2.133 & 3.750 & 117 & 0.070 & 0.389 \\
\hline 38 & 0.536 & 0.583 & 78 & 0.757 & 0.974 & 118 & 0.070 & 5.829 \\
\hline 39 & 0.796 & 0.815 & 79 & 0.070 & 0.812 & & & \\
\hline 40 & 1.195 & 1.305 & 80 & 3.710 & 4.072 & & & \\
\hline
\end{tabular}


Table 2 Top 20 important nodes ranked with the four evaluation methods

\begin{tabular}{ccccc|ccccc}
\hline \hline Ranking & $\begin{array}{c}\text { EBT } \\
\text { method }\end{array}$ & $\begin{array}{c}\text { PR } \\
\text { method }\end{array}$ & $\begin{array}{c}\text { LR } \\
\text { method }\end{array}$ & $\begin{array}{c}\text { ELR } \\
\text { method }\end{array}$ & Ranking & $\begin{array}{c}\text { EBT } \\
\text { method }\end{array}$ & $\begin{array}{c}\text { PR } \\
\text { method }\end{array}$ & $\begin{array}{c}\text { LR } \\
\text { method }\end{array}$ & $\begin{array}{c}\text { ELR } \\
\text { method }\end{array}$ \\
\hline 1 & 65 & 110 & 66 & 80 & 11 & 17 & 112 & 23 & 37 \\
2 & 68 & 111 & 89 & 69 & 12 & 37 & 9 & 65 & 15 \\
3 & 80 & 89 & 69 & 89 & 13 & 23 & 30 & 27 & 5 \\
4 & 38 & 87 & 80 & 92 & 14 & 70 & 8 & 9 & 66 \\
5 & 30 & 80 & 26 & 118 & 15 & 96 & 77 & 92 & 65 \\
6 & 81 & 69 & 10 & 77 & 16 & 94 & 55 & 64 & 56 \\
7 & 100 & 66 & 25 & 49 & 17 & 75 & 37 & 103 & 110 \\
8 & 49 & 26 & 5 & 100 & 18 & 24 & 59 & 111 & 105 \\
9 & 77 & 10 & 8 & 12 & 19 & 82 & 43 & 87 & 61 \\
10 & 69 & 86 & 49 & 17 & 20 & 64 & 34 & 91 & 19 \\
\hline \hline
\end{tabular}

For further investigation of the proposed ELR method, the EBT method in [10], the electrical PR method in [14], and basic LR method in [15] are also introduced and carried out on the studied power system for in-depth analysis and comparisons, and the rankings of top 20 important nodes obtained with the four evaluation methods are shown in Table 2. Furthermore, in order to demonstrate the validity and effectiveness of the proposed ELR, the important nodes from different methods are attacked to test the amount of load loss and network integrity based on the cascading failure model under interval uncertainties. Five attack modes, including random node attack, EBT node attack, PR node attack, LR node attack, and ELR node attack, are adopted to simulate the system attacks and assess the power grid failure consequences [8]. The random node attack is to attack a node, and then gradually attack more nodes in the power grid randomly. The other attack modes would deliberately attack the critical node with the highest importance, and then attack the nodes according to their rankings obtained from the corresponding methods in Table 2, respectively.

In this paper, four vulnerability metrics in [8], including average load loss, maximum amount of load, network transmission efficiency, and system connectivity, are used to further measure and compare the performances of different evaluation methods.

(1) Average load loss $L L$ : This index is to measure the average loss of load caused by the cascading failure when the top important nodes are attacked, as follows,

$$
L L=\frac{\sum_{i=1}^{N_{\mathrm{T}}} P_{\mathrm{LL} i}}{N_{\mathrm{T}}}
$$

where $N_{\mathrm{T}}$ is the number of top important nodes obtained with the evaluation method, and it is set to 20 in this case study; $P_{\mathrm{LL} i}$ represents the total load loss when the ith important node is attacked, and it can be calculated by the cascading failure model in Fig. 1 . 
(2) Maximum amount of load $R$ : The fundamental function of power grids is to provide sufficient and reliable power supply for the load demand. This index is to measure the maximum amount of load which can be supported by the existing power network, and it is an important index to evaluate the vulnerability of large-scale power grids, as follows,

$$
R=\frac{P_{\text {load,max }}}{P_{\text {load0 }}}
$$

where $P_{\text {loado }}$ denotes the total initial load of the power system; $P_{\text {load,max }}$ is the maximum amount of load which can be supported by the system after the attack, and it can also be determined by the sum of the maximum capacities of all the sub-systems after the islanding.

(3) Network transmission efficiency $T E$ : This index is to assess the system transmission performance, and it can be reflected on the shortest paths between the pairs from generator nodes to load nodes. Hence, the definition of $T E$ is the sum of all the reciprocals of the lengths of the shortest transmission paths, and the sum can further be normalized using the network transmission efficiency before the attacks as the base. It can be mathematically presented as,

$$
T E=\frac{\sum_{i \in S_{\mathrm{G}}, j \in S_{\mathrm{D}}} \frac{1}{d_{i j}}}{\sum_{i \in S_{\mathrm{G} 0}, j \in S_{\mathrm{D} 0}} \frac{1}{d_{i j 0}}}
$$

where $S_{\mathrm{G}}$ and $S_{\mathrm{D}}$ represent the sets of generator nodes and load nodes, respectively; $d_{i j}$ indicates the length of shortest transmission path from generator node $i$ to load node $j ; S_{\mathrm{G} 0}, S_{\mathrm{D} 0}$ and $d_{i j 0}$ are the corresponding sets or parameters for the power grid before the attack.

(4) System connectivity $Q$ : This index is to assess whether the power network can maintain its normal power supplies with system integrity, especially when the islanding occurs after cascading failures due to the node attacks. It can be calculated by the number of nodes in the largest connected sub-system after each attack, as follows,

$$
Q=\frac{\max \left(\operatorname{sum}\left(S_{\mathrm{B} m}\right)\right)}{N}, \quad m=1,2, \cdots, M_{\text {sub }}
$$

where $S_{\mathrm{B} m}$ represents the set of nodes in the sub-system $m$ after the islanding; $M_{\text {sub }}$ is the number of subsystems; $N$ is the number of nodes in the system before the attack.

A comparative study on the average loss of load obtained with the five attack modes is presented in Table 3. When the top 20 important nodes ranked by ELR are attacked, the average value of load loss caused by cascading failures is $473.08 \mathrm{MW}$, which is the highest loss among five node attacks. The resulting statistics demonstrate that the attacks on the critical nodes from ELR method will lead to more load blackouts than other evaluation methods. As the uncertainties of renewable energy and the transmis- 
Table 3 A comparison study on the load loss from top 20-node attacks

\begin{tabular}{cc}
\hline \hline Attack method & Average loss of load (MW) \\
\hline Random node attack & 119.10 \\
EBT node attack & 293.53 \\
PR node attack & 333.88 \\
LR node attack & 255.50 \\
ELR node attack & $\mathbf{4 7 3 . 0 8}$ \\
\hline
\end{tabular}

Fig. 5 illustrates the vulnerability of power grids when the system nodes are gradually attacked. The maximum amount of load which could be supported by the existing power network only decreases by approximately $20 \%$ after 20 nodes are randomly attacked consecutively, which indicates the power grid is more robust to random node attack compared with the deliberate attacks. Also, the maximum amount of load index with the other intentional attack modes decline remarkably, and ELR node attack is the most destructive as the system topological structure and load losses in the cascading failure scenarios are taken into consideration. Taking the No. 92 node in the ELR method as an example, since most of its connected nodes are load nodes, the adjacent loads will be greatly affected when the No. 92 node is attacked. As a result, cascade failures are likely to happen along with the decrease of the maximum amount of load while other methods fail to identify this node. It can also be found that the PR and LR node attacks can result in the severe system destruction after the top 20 nodes attacked.

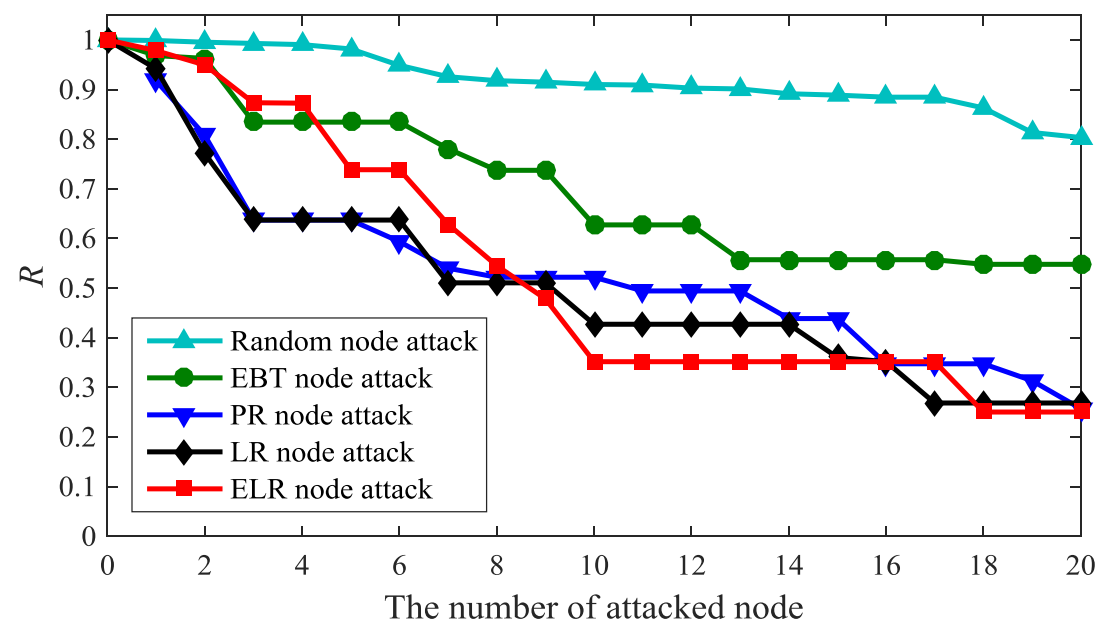

Fig. 5 Maximum amount of load index for 118-bus power system under node attacks 


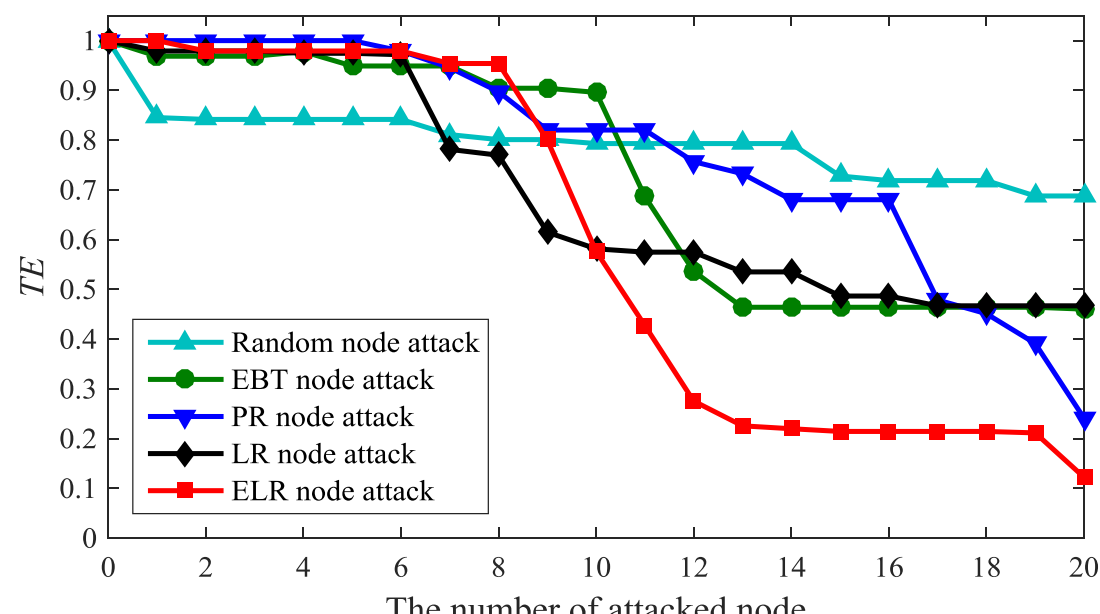

Fig. 6 Network transmission efficiency index for 118-bus power system under node attacks

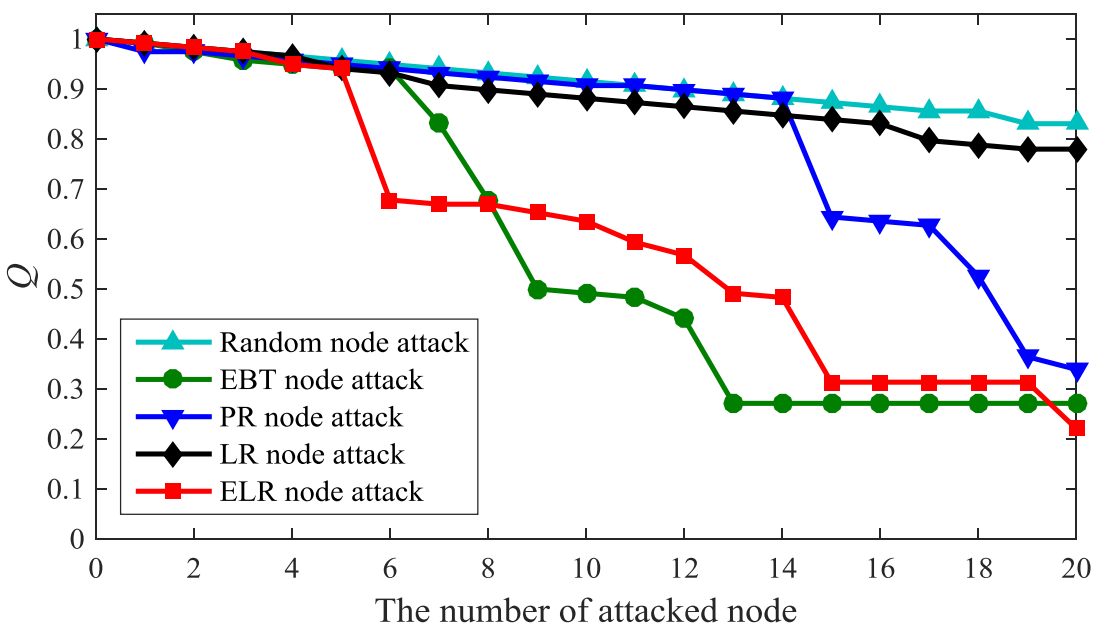

Fig. 7 System connectivity index for 118-bus power system under node attacks

In Fig. 6, the network transmission efficiency index decreases slightly during the random node attack scenarios, while this index declines abruptly under other intentional attacks. ELR node attack inflicts the maximum damage to network transmission performance, and the $T E$ decreases nearly to 0.1 with power network collapse after top 20 important nodes are attacked consecutively. It can be observed that when the boundary nodes between the two regions, such as No. 118 node from ELR method, is attacked, the system generation capacity will significantly decrease, contributing to the deterioration of network transmission efficiency. On the other hand, it can be seen from Fig. 7 that, the system connectivity of the power grid declines slightly during the attack of first 5 nodes, indicating that there is no isolated island disconnected from the grid. Among the five attack modes, ELR node attack has the severest impact on destroying the integrity of power grids than other attacks after the corresponding 20 critical nodes are attacked, while the EBT evaluation method can provide the top superior nodes on the system connectivity 
1 index. This is because the nodes with high electrical betweenness values in the EBT method are usually

2 the bridges connecting two sub-systems, and the nodes acting as bridges between sub-systems are usually

3 playing vital roles in the integrity of power grids. On the other hand, the ELR method considers the nodes 4 with a number of in-linked and out-linked nodes as the important nodes, and thus the performance of the 5 proposed method is not as well as the EBT method on the system connectivity metric.

\section{B. Study on the Western Liaoning Power Grid of China}

For the in-depth investigation of the proposed ELR in a realistic simulation, the power transmission network of the 54-bus Western Liaoning Power Grid of China, as shown in Fig. 8, is employed for further analysis and discussion. The studied power system contains five control areas, Chaoyang, Huludao, Jinzhou, Panjin and Fuxin. Its 500/220kV transmission system can be modeled as a complex network with

1154 nodes and 86 edges. The ranking of top 20 important nodes obtained with the ELR method is shown in Table 4 and marked in Fig. 8. It can be found that, the most of top nodes are the generator or substation nodes at the voltage level of $500 \mathrm{kV}$ and also form the long-distance and large-capacity transmission lines from the power generation center and load centers. The attacks on these critical nodes would cause huge power shortage and system frequency drops, resulting in a serious threat to power grid stability and security. The rest of nodes in Table 4 are boundary nodes to ensure the system connectivity, and the studied power grid would split into scattered control areas once these nodes are attacked. The results also demonstrate the proposed ELR measure can accord with the actual power system operation conditions.

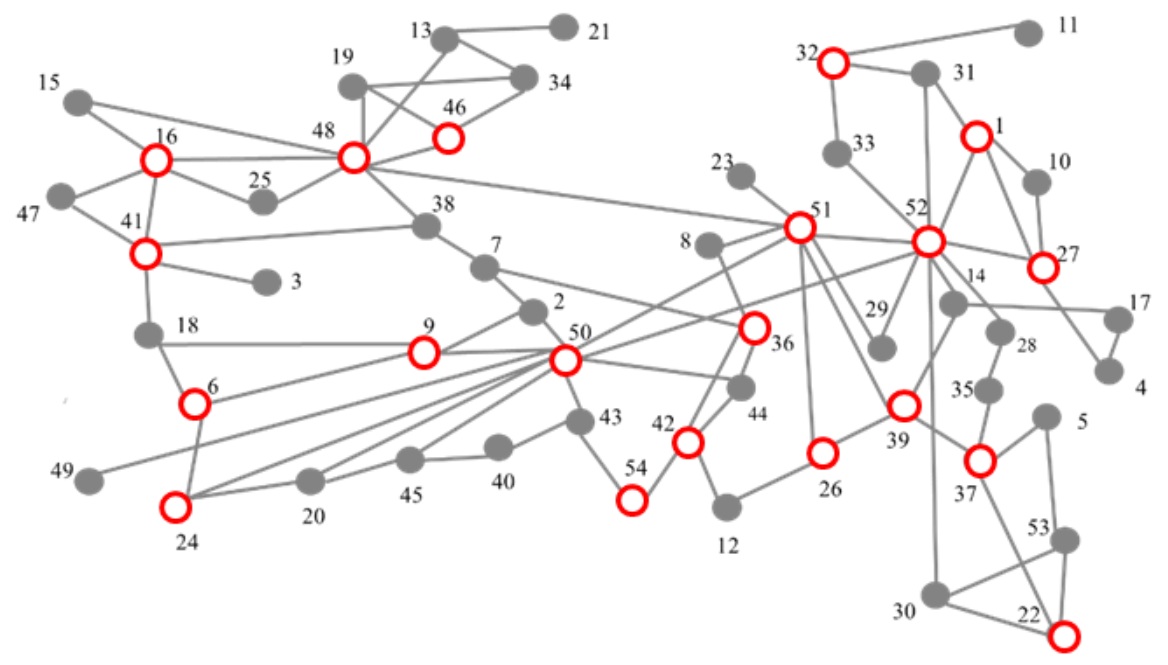

Fig. 8 Network topology of the Western Liaoning Power Grid of China 
Table 4 Top 20 important nodes and corresponding ELR intervals of the Western Liaoning power grid

\begin{tabular}{ccccc|ccccc}
\hline \hline Ranking & Node No. & Bus Name & $\begin{array}{c}E L R^{\mathrm{L}} \\
\text { value }\end{array}$ & $\begin{array}{c}E L R^{\mathrm{U}} \\
\text { value }\end{array}$ & Ranking & Node No. & Bus Name & $\begin{array}{c}E L R^{\mathrm{L}} \\
\text { value }\end{array}$ & $\begin{array}{c}E L R^{\mathrm{U}} \\
\text { value }\end{array}$ \\
\hline 1 & 50 & Shaheying & 2.052 & 3.358 & 11 & 16 & Jianping & 1.185 & 1.323 \\
2 & 51 & Dongjia & 1.272 & 3.284 & 12 & 27 & Qingduizi & 0.643 & 1.729 \\
3 & 52 & Beining & 1.515 & 2.849 & 13 & 42 & Nanshan & 0.081 & 2.237 \\
4 & 48 & Yannan & 1.139 & 2.065 & 14 & 46 & Longcheng & 0.464 & 1.794 \\
5 & 41 & Kazuo & 1.532 & 1.558 & 15 & 22 & Liaohe & 1.055 & 1.193 \\
6 & 36 & Jinzhou & 1.117 & 1.967 & 16 & 24 & Suizhong & 0.325 & 1.899 \\
7 & 39 & Shuguang & 0.853 & 2.115 & 17 & 32 & Songtao & 0.985 & 1.225 \\
8 & 9 & Xuejia & 1.422 & 1.538 & 18 & 54 & Gangwan & 0.871 & 1.003 \\
9 & 37 & Xinglongtai & 1.011 & 1.851 & 19 & 26 & Linghe & 0.808 & 0.964 \\
10 & 1 & Shuiquan & 0.398 & 2.262 & 20 & 6 & Madaoling & 0.810 & 0.812 \\
\hline \hline
\end{tabular}

Complex power grids have long-distance transmission lines and key nodes, such as generator nodes with lots of outgoing lines. When the power faults occur on these nodes, the cascading failures usually trigger and may further develop into large-scale blackouts [30]. Moreover, the uncertainties of high-penetration REGs would further result in the severe vulnerability issues of power systems, which cannot be evaluated using conventional methods due to uncertainties in the power flow distribution. Therefore, the weighted distribution strategy and interval uncertainties of REGs are considered in the proposed ELR method to evaluate the important nodes in the power system. Compared with the previous methods including EBT, PR and LR methods on various vulnerability metrics, the proposed method is valid and effective to identify the critical nodes of power grids with REGs, as the ELR node attack can outperform other deliberate node attacks on the loss of load, maximum supported load, network transmission efficiency and power system connectivity.

\section{Discussion}

It can be found from Fig. 1 that, the node importance of power grids can be ranked based on the load loss of cascading failures after the corresponding node attack, and the nodes with larger loss of load tend to be more important than others. On the other hand, the interval uncertainty analysis and modelling are performed for the outputs of REGs and the iteration strategy of ELR value function to enhance the identification accuracy of system important nodes. Thus, the following three node attack schemes are considered for further comparison and discussion: 1) Node attack scheme I is to attack the important nodes ranked by the average load loss as shown in Fig. 4; 2) Node attack scheme II implements the ELR node attack without considering the interval power flow and interval ELR values caused by variable WTG and PV outputs; 3) Node attack scheme III is the proposed ELR method considering the uncertainties of REGs. 
Table 5 Top 20 important nodes ranked with node attack schemes I-III

\begin{tabular}{cccc|cccc|cccc}
\hline \hline Ranking & $\begin{array}{c}\text { Node } \\
\text { attack I }\end{array}$ & $\begin{array}{c}\text { Node } \\
\text { attack II }\end{array}$ & $\begin{array}{c}\text { Node } \\
\text { attack III }\end{array}$ & Ranking & $\begin{array}{c}\text { Node } \\
\text { attack I }\end{array}$ & $\begin{array}{c}\text { Node } \\
\text { attack II }\end{array}$ & $\begin{array}{c}\text { Node } \\
\text { attack III }\end{array}$ & Ranking & $\begin{array}{c}\text { Node } \\
\text { attack I }\end{array}$ & $\begin{array}{c}\text { Node } \\
\text { attack II }\end{array}$ & $\begin{array}{c}\text { Node } \\
\text { attack III }\end{array}$ \\
\hline 1 & 89 & 69 & 80 & 8 & 59 & 89 & 100 & 15 & 54 & 30 & 65 \\
2 & 69 & 80 & 69 & 9 & 118 & 5 & 12 & 16 & 110 & 54 & 56 \\
3 & 49 & 17 & 89 & 10 & 116 & 77 & 17 & 17 & 12 & 70 & 110 \\
4 & 42 & 49 & 92 & 11 & 68 & 37 & 37 & 18 & 15 & 23 & 105 \\
5 & 25 & 32 & 118 & 12 & 80 & 92 & 15 & 19 & 56 & 59 & 61 \\
6 & 92 & 12 & 77 & 13 & 90 & 56 & 5 & 20 & 37 & 19 \\
7 & 100 & 100 & 49 & 14 & 85 & 94 & 66 & & 19 & \\
\hline \hline
\end{tabular}

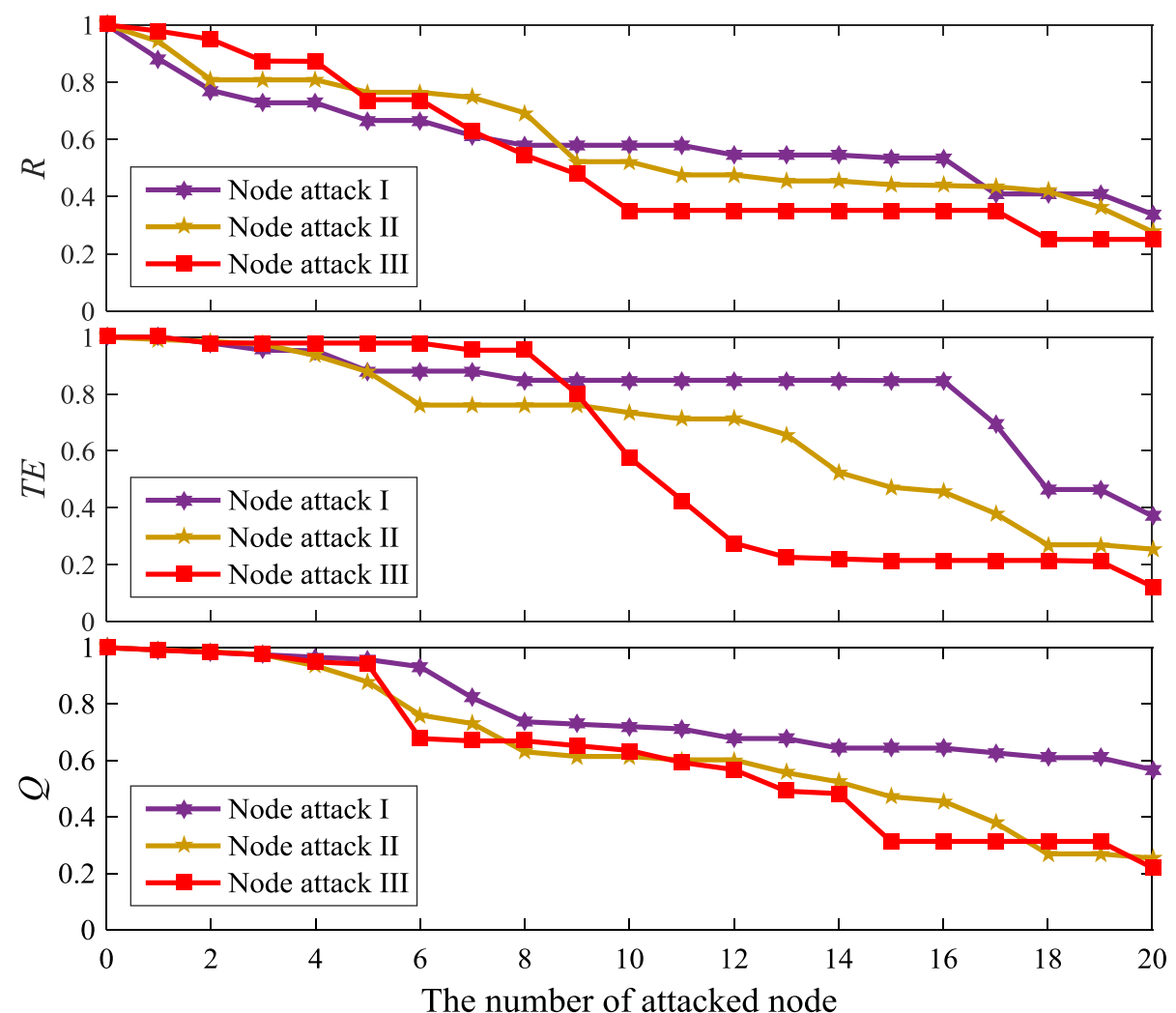

Fig. 9 Vulnerability metrics for 118-bus power system under node attack schemes I-III

With the node attack schemes I-III, the rankings of top 20 important nodes and comparative simulations on various vulnerability metrics are shown in Table 5 and Fig. 9, respectively. In this study, the average load loss under node attack schemes I and II are 648.42 MW and 460.30 MW, respectively. It can be found from comparative results that, although the loss of loads with node attack scheme I is larger than that of ELR method in Table 3, the proposed scheme III can outperform other schemes on the maximum

9 supported load, network transmission efficiency and power system connectivity. Furthermore, as the in10 termittency and volatility of REGs would increase the risk of transmission line overload and load shedding, 11 the scheme II exhibits poor performance on the average load loss and network transmission efficiency 12 because of ignoring the uncertainties of wind and solar energy generations. Consequently, it can be con- 
1 cluded that the proposed ELR scheme can perform the best with respect to the overall performance of

2 various vulnerability metrics.

\section{Conclusions}

In this paper, an electrical LeaderRank method is proposed to evaluate and rank the nodal importance of complex power grids with high penetration of variable renewables. Comparative studies on various system vulnerability measures demonstrated the superiority of the proposed ELR and confirmed its capability to cope with the identification of system important nodes under a stochastic environment. The conclusions of this investigation are summarized as follows: 1) The uncertainties in the nodal power injections from intermittent and volatile REGs may give rise to cascading overload failures, and the proposed ELR can effectively handle these uncertainties based on the interval power flow and interval ELR values; 2) It can be concluded from comparative results that the proposed ELR can observably outperform the other node evaluation methods, and provides satisfactory performance on various vulnerability metrics, especially on maximum amount of load and network transmission efficiency; 3) Simulation studies on the Western Liaoning Power Grid of China indicate the proposed ELR is promising and can be readily applied for vulnerability assessment of practical power systems with high-penetration renewables.

It should be pointed out that the DC power flow calculation is adopted to simulate cascading overload failures because of its linearized model and efficient computation. However, the reactive power and voltage information cannot be included in the DC-OPF model, and hence the blackout events caused by voltage collapse are not involved in this study. Further on-going research would focus on the probabilistic AC power flow model to analyze the blackout mechanism resulted from voltage stability problem.

\section{Acknowledgment}

This work was jointly supported by the National Natural Science Foundation of China (51507056), Hunan Strategic Industries Scientific and Technological Project under Grant 2017GK4028, and the Hunan Natural Science Foundation of China (2017JJ3019).

\section{References}

[1] Nedic D P, Dobson I, Kirschen D S, et al. Criticality in a cascading failure blackout model. International Journal of Electrical Power \& Energy Systems, 2006, 28(9): 627-633.

[2] Allen E H, Stuart R B, Wiedman T E. No light in August: power system restoration following the 2003 North American blackout. IEEE Power and Energy Magazine, 2014, 12(1): 24-33. 
[3] Yan J, He H, Sun Y. Integrated security analysis on cascading failure in complex networks. IEEE Transactions on Information Forensics and Security, 2014, 9(3): 451-463.

[4] Huang Z, Wang C, Zhu T, et al. Cascading failures in smart grid: Joint effect of load propagation and interdependence. IEEE Access, 2015, 3: 2520-2530.

[5] Tajer A, Dobson I, Kar S, et al. Guest editorial the theory of complex systems with applications to smart grid operations. IEEE Transactions on Smart Grid, 2016, 7(4): 1949-1950.

[6] Gupta S, Kazi F, Wagh S, et al. Analysis and prediction of vulnerability in smart power transmission system: A geometrical approach. International Journal of Electrical Power \& Energy Systems, 2018, 94: 77-87.

[7] Bompard E, Pons E, Wu D. Extended topological metrics for the analysis of power grid vulnerability. IEEE Systems Journal, 2012, 6(3): 481-487.

[8] Mei S, Zhang X, Cao M. Power grid complexity. Springer Science \& Business Media, 2011.

[9] Kivimäki I, Lebichot B, Saramäki J, et al. Two betweenness centrality measures based on Randomized Shortest Paths. Scientific reports, 2016, 6: 19668.

[10] Wang K, Zhang B, Zhang Z, et al. An electrical betweenness approach for vulnerability assessment of power grids considering the capacity of generators and load. Physica A: Statistical Mechanics and its Applications, 2011, 390(23): 4692-4701.

[11] Wang Z, Scaglione A, Thomas R J. Electrical centrality measures for electric power grid vulnerability analysis. In: 49th IEEE Conference on Decision and Control (CDC), 2010: 5792-5797.

[12] Xiao Q. A method for measuring node importance in hypernetwork model. Research Journal of Applied Sciences, Engineering and Technology, 2013, 5(2): 568-573.

[13] Page L, Brin S, Motwani R, et al. The PageRank citation ranking: Bringing order to the web. Stanford InfoLab, 1999.

[14] Li C, Liu W, Cao Y, et al. Method for evaluating the importance of power grid nodes based on PageRank algorithm. IET Generation, Transmission \& Distribution, 2014, 8(11): 1843-1847.

[15] Lv L, Zhang Y C, Yeung C H, et al. Leaders in social networks, the delicious case. PloS one, 2011, 6(6): e21202.

[16] Xu S, Wang P. Identifying important nodes by adaptive LeaderRank. Physica A: Statistical Mechanics and its Applications, 2017, 469: 654-664.

[17] Ma Z, Chen H, Chai Y. Analysis of voltage stability uncertainty using stochastic response surface method related to wind farm correlation. Protection and Control of Modern Power Systems, 2017, DOI: 10.1186/s41601 -017-0051-3.

[18] Volkanovski A. Wind generation impact on electricity generation adequacy and nuclear safety. Reliability Engineering \& System Safety, 2017, 158: 85-92.

[19] Athari M H, Wang Z. Impacts of wind power uncertainty on grid vulnerability to cascading overload failures. IEEE Transactions on Sustainable Energy, 2018, 9(1): 128-137.

[20] Xu L, Ruan X, Mao C, et al. An improved optimal sizing method for wind-solar-battery hybrid power system. IEEE Transactions on Sustainable Energy, 2013, 4(3): 774-785. 
[21] Vaccaro A, Cañizares C A, Bhattacharya K. A range arithmetic-based optimization model for power flow analysis under interval uncertainty. IEEE Transactions on Power Systems, 2013, 28(2): 1179-1186.

[22] Levin V I. Comparison of interval numbers and optimization of interval-parameter systems. Automation and Remote Control, 2004, 65(4): 625-633.

[23] Zhou B, Xu D, Li C, et al. Multiobjective generation portfolio of hybrid energy generating station for mobile emergency power supplies. IEEE Transactions on Smart Grid, 2017, DOI: 10.1109/TSG.2017.2696982.

[24] Kargarian A, Mohammadi J, Guo J, et al. Toward distributed/decentralized DC optimal power flow implementation in future electric power systems. IEEE Transactions on Smart Grid, 2018, 9(4): 2574-2594.

[25] Bao H, Wei H, Guo X. Interval optimal power flow calculation for AC/DC power system considering renewable energy source. Proceedings of the CSEE, 2015, 35(16): 4006-4014.

[26] Zhang C, Chen H, Guo M, et al. DC power flow analysis incorporating interval input data and network parameters through the optimizing-scenarios method. International Journal of Electrical Power \& Energy Systems, 2018, 96: 380-389.

[27] Chen C, Wang F, Zhou B, et al. An interval optimization based day-ahead scheduling scheme for renewable energy management in smart distribution systems. Energy Conversion and Management, 2015, 106: 584-596.

[28] Wu J, Watts D J. Small worlds: the dynamics of networks between order and randomness. ACM, 2002.

[29] Zhao X, Zhang X, He B. Study on self-organized criticality of China power grid blackouts. Energy Conversion and Management, 2009, 50(3): 658-661.

[30] Mei S, Ni Y, Wang G, et al. A study of self-organized criticality of power system under cascading failures based on AC-OPF with voltage stability margin. IEEE Transactions on Power Systems, 2008, 23(4): 17191726.

[31] Volkanovski A, Čepin M, Mavko B. Application of the fault tree analysis for assessment of power system reliability. Reliability Engineering \& System Safety, 2009, 94(6): 1116-1127.

[32] Zhang X, Zhan C, Chi K T. Modeling the dynamics of cascading failures in power systems. IEEE Journal on Emerging and Selected Topics in Circuits and Systems, 2017, 7(2): 192-204.

[33] Dobson I, Newman D E. Cascading blackout overall structure and some implications for sampling and mitigation. International Journal of Electrical Power \& Energy Systems, 2017, 86: 29-32.

[34] Mei S, He F, Zhang X, et al. An improved OPA model and blackout risk assessment. IEEE Transactions on Power Systems, 2009, 24(2): 814-823.

[35] Li Q, Zhou T, Lv L, et al. Identifying influential spreaders by weighted LeaderRank. Physica A: Statistical Mechanics and its Applications, 2014, 404: 47-55.

[36] Zimmerman, R. D., Murillo-Sanchez, C. E., Gan, D.: Matpower: a Matlab power system simulation package, Power Systems Engineering Research Center, Available: http://www.pserc.cornell.edu/matpower

[37] Wan C, Xu Z, Pinson P, et al. Probabilistic forecasting of wind power generation using extreme learning machine. IEEE Transactions on Power Systems, 2014, 29(3): 1033-1044. 